\title{
Measuring the Permeability of Vacuum Using a Smartphone
}

\section{Jarier Wannous ${ }^{\text {a }}$, Peter Horváth ${ }^{\text {b }}$}

${ }^{a}$ Comenius University in Bratislava, Faculty of Mathematics, Physics and Informatics, Department of Didactics in Mathematics, Physics and Informatics, jarier.wannous@fmph.uniba.sk, ${ }^{\mathrm{b}}$ Comenius University in Bratislava, Faculty of Mathematics, Physics and Informatics, Department of Didactics in Mathematics, Physics and Informatics, peter.horvath@fmph.uniba.sk

\begin{abstract}
The paper offers a few activities for high school students which use the magnetometer of a smartphone to measure the value of magnetic fields. The first part of the paper deals with finding the magnetometer of the used smartphone. Following is the first selection of activities which are focused on discovering the equation for measuring the magnetic field of coil with a negligible length, while the second selection of activities use the discovered equation to measure the permeability of vacuum and finally to measure the magnetic field of the earth. Sample results of the experiments are given, showing the accuracy and effectiveness of the conducted experiments. The activities offer teachers a novel way for teaching the equation for calculating the magnetic field of a coil, as well as measuring the permeability of vacuum in a classroom environment.
\end{abstract}

Keywords: magnetic field of coils, magnetic field of earth, permeability of vacuum, BYOD, student activities.

\section{Introduction}

Using a smartphone's sensors in physics experiments and in the classroom is not a new phenomenon. Many studies have shown the possibilities of using smartphone sensors in different experiments and activities (Sukariasih, Erniwati, Sahara, Hariroh, \& Fayanto, 2019). The surge of these kind of activities and experiments even led to the development of BYOD (Bring Your Own Device) classrooms, where smartphones have become an available tool for physics experiments (Parsons \& Adhikari, 2016). Similarly, many activities have been introduced using the magnetometer of a smartphone, Arribas for example uses the magnetometer to show the magnetic field of a bar magnet (Arribas, 
Escobar, Suarez, Najera, \& Beléndez, 2015) and Silva used it to show how the magnetic field of a solenoid changes with the numbers of turns it has (Silva, 2012).

In our paper we offer a series of activities using the magnetometer of a smartphone starting by experimentally discovering the equation for the magnetic field of a coil, measuring the permeability of vacuum and finally the value of Earth's magnetic field. Example results of the activities are given as well as a quick description of how the activities can be implemented in a high school classroom.

\section{The Magnetometer of a Smartphone}

In the following activities, the location of the magnetometer on the smartphone is important for acquiring an accurate result, that is why the first step is locating it. Students get a simple assignment: Locate the magnetometer on your phone. To find it, students will need two things, a magnet and a suitable compass app.
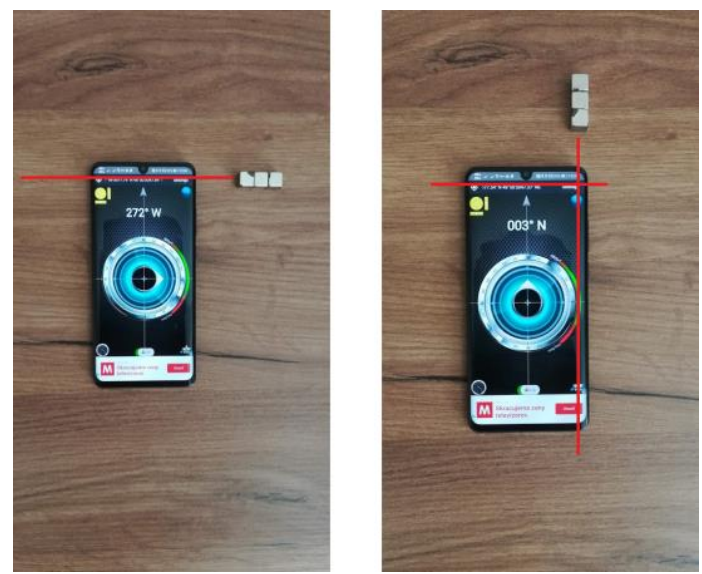

Fig. 1 Left: The magnet is moved next to the right side of the smartphone until the smartphone shows north to be to the right. Right: The magnet is moved above the upper edge of the smartphone until it shows north to be up. The location of the magnetometer is where the right and upper directions intersect.

Not all compass apps use the magnetometer of the smartphone to show North. Many use GPS in combination with the magnetometer in case there is a magnetic field disrupting the results from the magnetometer. As our goal is not to show the direction north, it is important to use a compass app which does not primarily rely on GPS. For this reason, we recommend the app Compass 360 Pro, which is freely available for android devices.

To find the location of the magnetometer, the magnet can be moved around the edges of the smartphone. If the magnet is moved near the right and upper edge of the smartphone, then 
the location of the magnetometer can be easily discerned when the compass shows that north is to the right, or up (figure 1).

\section{The Magnetic Field of a Coil}

The first experiment in the series is measuring the magnetic field of a coil. Specifically, a coil with a negligible length, i.e. its length is considerably smaller than its diameter. According to Halliday (2011), for a single turn in such a coil, the magnitude of the magnetic field inside the turn can be calculated as:

$$
B=\frac{\mu_{0} I}{2 R}
$$

In other words, the magnetic field is directly proportional to the intensity of current in the coil and indirectly proportional to its diameter. For a coil with a negligible length, the magnetic field is also directly proportional to the number of turns in the coil, bringing us to the following equation:

$$
B=\frac{\mu_{0} n I}{2 R}
$$

In the first experiment, students should arrive to the above-mentioned proportionalities empirically on their own using their smartphone. The first step in such an experiment, is the preparation of the coils. In our case, we have prepared seven coils in total. Four of them have the same diameter and different numbers of turns and four of them have the same number of turns and different diameters. The coils are home made using a wire fixed on a box as shown in figure 2 .

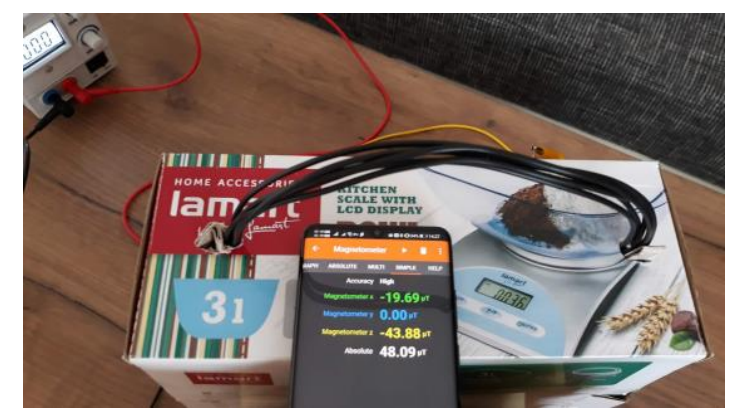

Fig. 2. Measuring in Phyphox using a smartphone's magnetometer. The magnetometer is approximately in the center of the coil, while the $y$-axis, the one measuring the magnetic field of the coil shows approximately $0 \mu T$ when the intensity of the current is $0 \mathrm{~A}$.

To verify any of the above-mentioned proportionalities, students should be able to measure the magnetic field of the coil. That can be done using the app Phyphox, which uses the 
magnetometer of the smartphone to measure the value of the magnetic field in three axes in real time. To measure the magnitude of the magnetic field inside the coil, the smartphone should be laid inside the coil so that the magnetometer, the location of which is known after the previous activity, is inside the coil. We also recommend that the magnetic field of the coil be directed east or west, so the magnetic field of the earth would not interfere with our measurement. This can be easily achieved using the magnetometer in Phyphox. The axis measuring the value of the magnetic field of the coil should show the value $0 \mu \mathrm{T}$ (figure 2 ).

When the instruments are set up as mentioned above, the coil is connected to a source of variable voltage and the intensity of current is measured using an ammeter, the experiment can start. By changing the voltage of the source, we measured the magnetic field and current on all seven homemade coils. Using the measured data, the graphs of magnetic field to current of all the coils. The graphs are presented in figures 3 and 4 .

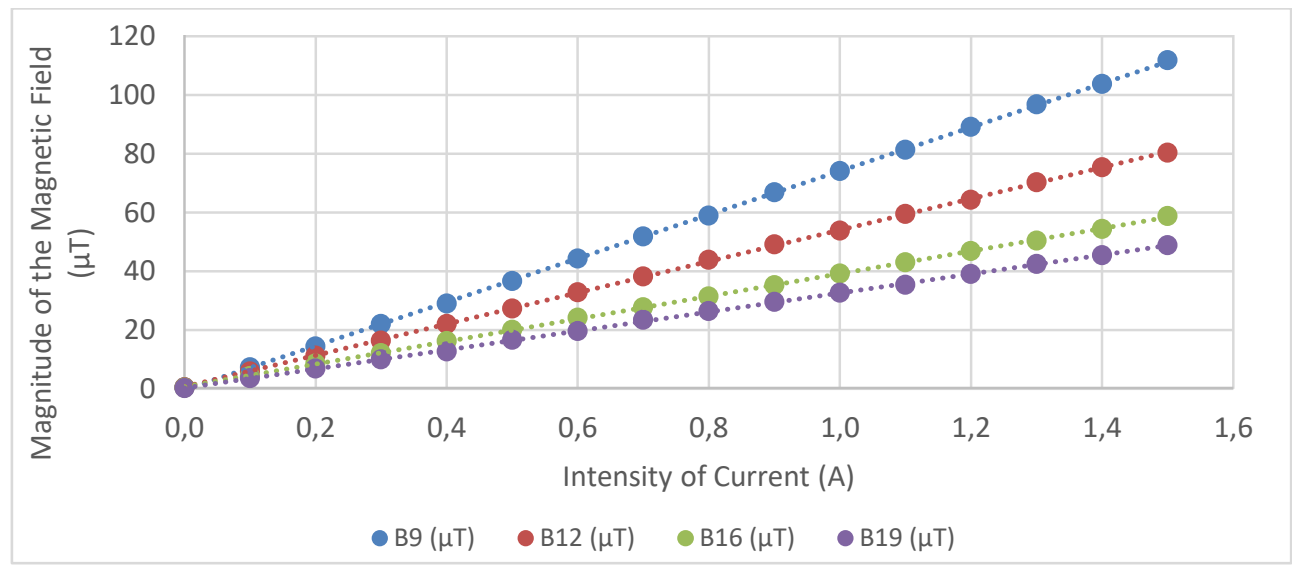

Fig. 3. The graphs of the magnitude of the magnetic field of a coil to the intensity of current for coils with the same number of turns, specifically 5 turns. The diameters of the coils are $9 \mathrm{~cm}$ (blue), $12 \mathrm{~cm}$ (pink), $16 \mathrm{~cm}$ (green) and $19 \mathrm{~cm}$ (purple).

All the measurements show a direct proportionality between the intensity of current and the magnitude of the magnetic field of the coil. Also, figure 4 implies an indirect proportionality between the diameter of the coil and the magnitude of the magnetic field, while figure 5 implies a direct proportionality between the number of turns and the magnitude of the magnetic field. These results agree with the equation (2) presented above.

To conduct this experiment in a classroom, the students can be first directed to predict the physical quantities that the magnetic field of the coil is dependent upon as well as the relationship between those quantities and the magnitude of the magnetic field. We expect that students, based on experience with a current carrying wire, will predict that the created magnetic field will be connected to the intensity of the current. Also, it should not be a huge leap for them to predict the relationship between the number of turns and the diameters of 
the coils and their magnetic fields. Even if all physical quantities are not predicted, the experiment can be conducted.

The student then divide into small groups. Each group will verify the relationship between the intensity of current and the magnitude of the magnetic field. When their results show a direct proportionality, they can compare their results together. As each group has a different coil, they should discuss the differences between their coils, leading them to prepare figures similar to figures 4 and 5 and discover the effect of the number of turns and the diameter of the coil.

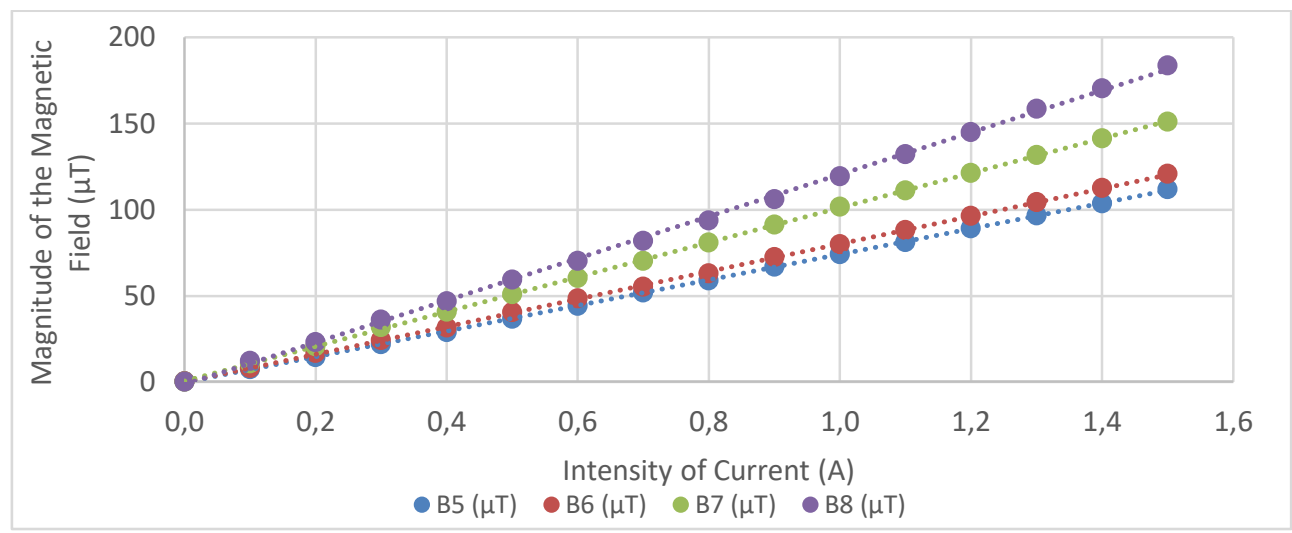

Fig. 4. The graphs of the magnitude of the magnetic field of a coil to the intensity of current for coils with the same diameter, specifically $9 \mathrm{~cm}$. The number of turns of the coils are 5 (blue), 6 (pink), 7 (green) and 8 (purple).

\section{The Permeability of Vacuum}

When considering equation (2), we can see that the slope of a graph of the magnitude of magnetic field to the intensity of current can be determined as following:

$$
k=\frac{\mu_{0} n}{2 R}
$$

meaning that permeability can be calculated as following:

$$
\mu_{0}=\frac{k 2 R}{n}
$$

Using a linear fit on the data from our previous experiment, the slopes of the graphs were acquired, and they are presented in table 1. The slopes are then used to measure the permeability of vacuum (air). The measured permeability is therefore:

$$
\mu_{0}=(1.277 \pm 0.049) \cdot 10^{-6} H \cdot m^{-1}
$$


While the difference between some of our values and the real value of permeability reached up to $9 \%$, the data are still acceptable for a classroom environment, especially as there are several factors affecting the results of the experiment. For example, a small tilt of the coil, an imprecise shape of the coil or the positioning of the magnetometer. Also, the coils with fixed diameter can present a slight problem for the experiment as it is much easier to prepare coils with a random diameter than with a fixed one. Therefore, mistakes in the diameter can easily reach 5\% and higher, affecting the results of the experiment.

Table 1. Measurement of permeability using the slopes of the graphs of the value of magnetic field to the intensity of current

\begin{tabular}{|cccc|}
\hline Number of turns $-\boldsymbol{n}$ & $\begin{array}{c}\text { Diameter of coil }- \\
2 \boldsymbol{R}(\mathbf{m})\end{array}$ & $\begin{array}{c}\text { Slope } \boldsymbol{k} \\
\left(. \mathbf{1 0}^{-\mathbf{6}} \mathbf{T} \cdot \mathbf{A}^{-\mathbf{1}}\right)\end{array}$ & $\begin{array}{c}\boldsymbol{\mu}_{0} \\
\left(.10^{-6} \mathbf{H}^{-\mathbf{1}}\right)\end{array}$ \\
\hline $\mathbf{5}$ & 0.19 & 32.3 & 1.23 \\
\hline $\mathbf{5}$ & 0.16 & 38.5 & 1.23 \\
\hline $\mathbf{5}$ & 0.12 & 53.4 & 1.28 \\
\hline $\mathbf{5}$ & 0.09 & 74.4 & 1.34 \\
\hline $\mathbf{6}$ & 0.09 & 80.2 & 1.20 \\
\hline $\mathbf{7}$ & 0.09 & 101 & 1.29 \\
\hline $\mathbf{8}$ & 0.09 & 122 & 1.37 \\
\hline
\end{tabular}

In the classroom, while the students can see that the value of the magnetic field is dependent on the intensity of the current, number of turns and the diameter of the coil, the teacher can argue that the students need to make sure that there are no other physical quantities missing. This could lead to using the measured slopes, eliminating the effects of the number of turns and the diameter. The students should get the unknown constant, which the teacher can then introduce as the permeability of vacuum, leading to a discussion about permeability of materials and magnetism in general. Also, the results can lead the students to arrive to equation (2) to calculate the value of the magnetic field of a coil with a negligible length.

\section{The Magnetic Field of Earth}

As students have now arrived at equation (2) and are able to calculate the magnetic field of a coil with a negligible length, this can be used to measure the magnetic field of the earth. To measure the magnetic field of it can be compared with a known magnetic field using a compass, or a compass app in our case. The method itself is not novel. For example, Amiri (2004) and Stewart (2000) compared the magnetic field of the earth with a solenoid, 
Williams (2014) compared it to a Helmholtz coil and Lunk (2011) compared it to the magnetic field of a long straight wire.

In the case of our experiment, we compare it with the magnetic field of a coil with a negligible length. The reason for using this coil is twofold: Such a coil is easily made at home and the equation for its magnetic field has been discovered experimentally. This method is also described in detail by Ganci (2004). In the experiment, instead of a compass, we use the previously discussed app Compass 360 Pro. The coil is set up so its magnetic field will be perpendicular to the magnetic field of the earth. When current goes through the coil, the magnetic fields of the coil and the earth will add up (figure 5) so the compass will show north at an angle $\varphi$. Knowing the value of the magnetic field of the coil $B_{C}$, the magnetic field of the Earth can be easily calculated as:

$$
B_{E}=\frac{B_{C}}{\tan \varphi}
$$

Using the magnetometer of a smartphone allows for a more accurate measurement of the angle than with a regular compass. Using this method we have measured the tangential component of the magnetic field of the earth to be $(20.5 \pm 0.71) \cdot 10^{-6} T$ which is a typical value of the tangential component of the magnetic field of earth at our position in Trenčin, Slovakia.
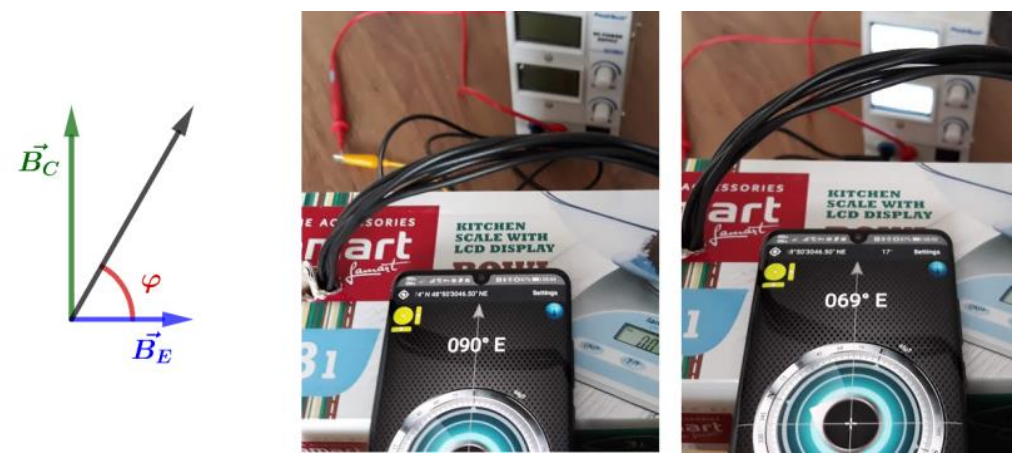

Fig. 5. Left: The addition of the magnetic fields of earth and the coil. Center: The setup of the compass at a $90^{\circ}$ angle to the magnetic field of the coil. Right: The change of the angle when current is flowing through the coil.

\section{Conclusion}

In this paper, we have presented activities using the magnetometer of a smartphone to discover the equation for calculating the magnetic field of a coil with a negligible length, to measure the permeability of vacuum and to measure the tangential component of the 
magnetic field of the Earth. The data acquired in the experiments are fully compatible with the needs of a basic physics course and therefore can be very useful for physics teachers. This is especially true as the experiments can be focused on students making predictions and verifying them experimentally with little guidance from the teacher.

\section{Acknowledgments}

This paper was elaborated with the support of the project VEGA no. 1/0396/18.

\section{References}

Amiri, F., \& Jeffery, R. N. (2004, November). Simple Experiments to Study the Earth's Magnetic Field. The Physics Teacher, 42, 458-461.

Arribas, E., Escobar, I., Suarez, C. P., Najera, A., \& Beléndez, A. (2015, October). Measurement of the magnetic field of small magnets with a smartphone: a very economical laboratory practice for introductory physics courses. European Journal of Physics, 36(6), 1-11.

Ganci, S. (2004, July). A simple measurement of the Earth's magnetic field. European Journal of Physics, 25(4), 475-477.

Halliday, D., Resnick, R., \& Walker, J. (2011). Fundamentals of Physics (9th ed.). Cleveland: John Wiley \& Sons, Inc.

Lunk, B., \& Beichner, R. (2011, January). Exploring Magnetic Fields with a Compass. The Physics Teacher, 49, 45-48.

Parsons, D., \& Adhikari, J. (2016, April). Bring Your Own Device to Secondary School: The Perceptions of Teachers, Students and Parents. Electronic Journal of e-Learning, 14(1), 67-81.

Silva, N. (2012, September). Magnetic Field Sensor. The Physics Teacher, 50, 372-373.

Stewart, G. B. (2000, February). Measuring Earth's Magnetic Field Simply. The Physics Teacher, 38, 113-114.

Sukariasih, L., Erniwati, Sahara, L., Hariroh, L., \& Fayanto, S. (2019, October). Studies The Use Of Smartphone Sensor For Physics Learning. INTERNATIONAL JOURNAL OF SCIENTIFIC \& TECHNOLOGY RESEARCH, 8(10), 862-870.

Williams, J. E. (2014, April). Measuring Earth's Local Magnetic Field Using a Helmholtz Coil. The Physics Teacher, 52, 236-238. 